\title{
Study of pore fluid effect on the mobility of granular debris flows
}

\author{
Gordon G. D. Zhou, ${ }^{1, *}$, and Q. C. $\operatorname{Sun}^{2}$ \\ ${ }^{1}$ Key Laboratory of Mountain Hazards and Earth Surface Processes/Institute of Mountain Hazards and Environment, \\ Chinese Academy of Sciences (CAS), Chengdu, China \\ ${ }^{2}$ State Key Laboratory for Hydroscience and Engineering, Tsinghua University, Beijing, China
}

\begin{abstract}
Granular debris flows in nature are composed of a wide range of solids and viscous pore fluids, moving at high velocities down sloping channels. The pore fluids in a granular debris flow affect the interactions between the solid and fluid phases and thus govern the debris-flow mobility. Study of the pore fluid effect (i.e., excess pore water pressures correlated to solid structures, and the viscous shearing and dragging) is essential for understanding the high flow mobility of granular debris flows. This study critically reviews two dimensionless numbers with clear physical meanings, then demonstrates a new application of field monitoring data for identifying natural debris flows on large scales (i.e., surge and continuous debrisflows, respectively). This study illustrates that, the pore fluid viscous shearing stress dominates solid inertial stress due to solids collision. It is also found that different to continuous debris-flows, the high pore fluid pressures generated in surge debris-flow body dissipate quite slowly and mostly influence particle contact behaviour significantly. A new scientific criterion for identification of continuous and surge debris flow in nature can be given by this study.
\end{abstract}

\section{Introduction}

Debris flows occur when masses of poorly sorted sediment, agitated and saturated with water, surge down a slope in response to gravitational attraction [1]. Debris flows differ from rock avalanches and sediment-laden water floods in that both solid and fluid forces influence the motion of a debris flow and governs its rheological properties [1]. Stresses caused by solid particle collisions, contact friction, and solid-fluid interactions (e.g., buoyancy, drag, added mass, lift, and Basset, Faxen, and grain diffusion forces) generally govern the kinematic properties of debris flows [1-4].

When studying the rheological properties of natural debris flows, properly identifying the flow regimes is always the most important issue. Unfortunately, the classification of debris flows nowadays is generally based on empirical observations due to the complexity of the phenomena. For instance, the monitoring debris flows in China were classified into surge and continuous flows mostly based on the surge duration and empirical judgement, which is believed to be quite different for surge and continuous flows. However, sometimes the time difference is quite small and it is really difficult to identify whether the surge duration is relatively long or short. Pure empirical judgements can cause huge mistakes. However, Coussot and Meunier [5] demonstrated that the criterion used to distinguish phenomena of debris flows and other subaerial flows available vary and contradict one another, and they are incapable of covering all phenomena. Considering fine muddy debris flows (mudflows) are viscoplastic fluid flows, whose behaviour is dictated by the fine interstitial clay-water mixture, non-dimensional numbers for water flows are modified and extrapolated for muddy debris flows based on the concept of "global similarity" [6, 7]. It is noted that, different from mud flows, individual debris flows can contain grains that range from clay size to boulder size. Moreover, the sand, gravel, and larger grains compose most of the mass of debris flows and that silt and clay-sized grains which are the component of a slurry commonly constitute less than $10 \%$ of the mass $[1,8,9]$. The ability of debris-flow solids to exhibit dense, interlocked packings as well as loose, high-porosity packings has significant ramifications for mixture behavior, which varies markedly with subtle variations in solid volume fraction (concentration), shear rate, and grain size distribution [10]. Basic studies on the dynamics of solid-fluid two-phase flows are needed for the better understanding of mobility of debris flows.

Bagnold [11] reported that flows of solid-fluid mixtures can be divided into grain-inertial flows and macro-viscous flows. These flows depend on the relative significance of solid collisions and pore fluid viscosity, and a dimensionless number called the Bagnold number was developed for identifying flow regimes in this way. Particle collisions dominate in grain-inertia flows, which correspond to a large Bagnold number, and the momentum transfer and frequency of collisions are both proportional to the relative velocity difference between two neighbouring layers (shear rate). The corresponding stresses caused by collisions are proportional to the square of the shear rate. In macro-viscous flows, which correspond to a small Bagnold number, the effect of

Corresponding author: gordon@imde.ac.cn 
fluid viscosity is dominant. Obviously, then, dimensionless numbers are a good criterion for identifying flow regimes.

In this paper, we reinterpret the recorded field data of viscous debris flows in Dongchuan, China, and identify the different flowing regimes of natural granular debris flows using two dimensionless numbers based on the relative importance of solids collision, pore fluid viscous drag and pore fluid pressures. A uniform criterion which has clear physical meanings is developed to clarify different natural debris flows.

\section{Field study at the Dongchuan Debris Flow Observation and Research Station}

The Dongchuan Debris Flow Observation and Research Station (DDFORS), located in the transportation zone of the Jiangjia Ravine in the Dongchuan district, Yunnan Province of China (N26 $14^{\prime}$, E $\left.103^{\circ} 08^{\prime}\right)$, is a facility of the Institute of Mountain Hazards and Environment, Chinese Academy of Sciences [12]. Since the 1960s, the station has conducted continual observations of field debris flows occurring in the Jiangjia Ravine due to intense rainfalls. The observations at DDFORS usually focus on a straight valley that is $270 \mathrm{~m}$ long on a $3.4^{\circ}$ slope. The cross-section is rectangular, and instruments are installed on two banks for observing and recording flow properties (see Fig.1).

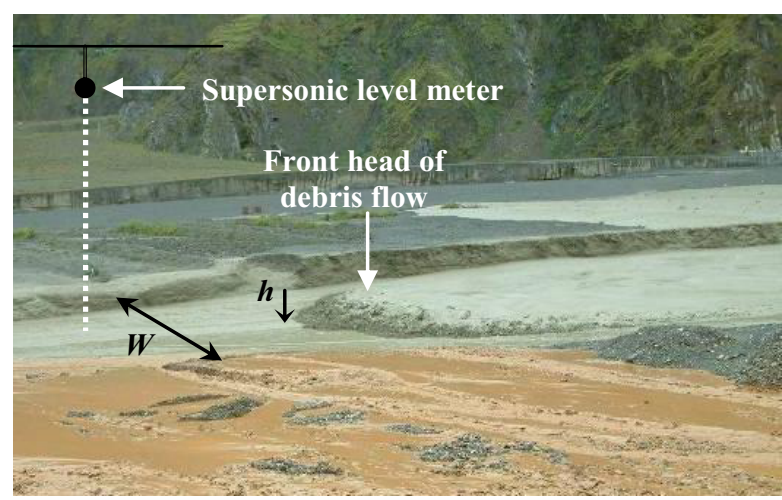

Fig. 1. Field observation area for natural debris flows in Jiangjia Ravine, China.

Two monitoring cross sections are marked along a straight channel to capture multiple debris-flow surges, with the predetermined distance $\Delta L$ used to measure the velocity of the surge front. The time for the surge front to pass through $\Delta L$ is recorded by a stopwatch and marked as $\Delta t$ for each debris flow, and the mean front velocity is then calculated as $U=\Delta L / \Delta t$. The channel width $W(m)$ and the ground level for each debris flow are determined by using cross section marks within the channel. A supersonic level meter installed above the front head measures the flow thickness $h(m)$, allowing the front discharge $Q$ to be calculated, where $Q=U \times W \times h$. The unit weight of each surge $\rho_{c}\left(\mathrm{~kg} / \mathrm{m}^{3}\right)$ is determined through direct sampling of the debris flow. Thus, the solid volume concentration $C_{S}$ can be calculated from $C_{S}=\left(\rho_{c^{-}} \rho_{w}\right) /\left(\rho_{S^{-}} \rho_{w}\right)$, where $\rho_{w}$ and $\rho_{S}$ are the unit weights of the water and solid material in the debris flow, respectively, and $\rho_{S}$ is generally taken to be $2750 \mathrm{~kg} / \mathrm{m}^{3}$. The solids discharge $Q_{S}(\mathrm{~kg} / \mathrm{s})$ is then calculated as $Q_{S}=Q \times \rho_{S} \times C_{S}$. Furthermore, as the duration of each surge is recorded, the total (or sediment) runoff of each surge can be determined, allowing the flows to be classified into surge flows and continuous flows. By empirical estimation, either the flow time of a continuous flow is quite long or the discharge is quite large. If there is an obvious flow break between two continual surges, or the sustained time is relatively short, the flow is treated as a surge flow [13,14,15]. Field observation data for the 1961-2000 period has been collected and edited by Kang et al. [14], Zhang and Xiong [13], and Kang et al. [15]. It is from this dataset that we can analyze the dynamics of debris flows.

\section{Dimensionless numbers with clear physical meanings for granular debris flows}

\subsection{Pore fluid viscous drag and solids collision: grain Reynolds number $\mathbf{N}_{\text {Rey }}$}

In fluid mechanics, the Reynolds number is well-known for identifying laminar and turbulent flows. For grainfluid mixture flows, the grain Reynolds number $N_{R e y}$ is applied to identify the effects of particle collisions and pore fluid viscosity. The definition of the grain Reynolds number is given in Eq. (1) (cf. Iverson 1997):

$$
N_{R e y}=\frac{T_{s(i)} / T_{f(q)}}{\frac{C_{s}}{1-C_{s}} \frac{\rho_{s}}{\rho_{f}}}=\frac{\rho_{f} \dot{\gamma} d^{2}}{\mu_{f}} \text { (1) }
$$

Eq. (1) clarifies the physical meaning of the grain Reynolds number $N_{\text {Rey }}$ : it is a ratio between the solid inertial stress due to particle collision, $T_{S(i)}$, and the fluid viscous shearing stress, $T_{f(q)}$,. The solid inertial stress may be estimated by $T_{s(i)} \sim C_{S} \rho_{s} \dot{\gamma}^{2} d^{2}$ (spherical grains of mean diameter $d$ and density $\rho_{s}$, sheared under gravity at a shear rate of $\dot{\gamma}=d U / d h$ ) and the fluid viscous shearing stress derived from Newton's law of viscosity is $T_{f(q)}=C_{f} \dot{\gamma} \mu_{f} \quad\left(C_{f}\right.$ is the pore fluid volume concentration equal to $1-C_{S}$ and $\mu_{f}$ is the viscosity of the pore fluid).

\subsection{Pore fluid pressure dissipation: $\mathbf{N}_{\mathbf{P}}$}

To distinguish the different debris flows based on the field observation data, a new criterion correlating with the flow duration and pore fluid pressure dissipation is developed. Reconsider the dimensionless number $N_{P}$ defined by Iverson et al. (2004)

$$
N_{p}=\frac{\sqrt{l / g}}{\mu_{f} h^{2} / k E}
$$

where $l$ is the avalanche length and can be calculated by $U \cdot t$ ( $\mathrm{t}$ is the surge duration), $k$ is the hydraulic permeability. The definition shows that $N_{P}$ is not only correlated to surge duration but also has clear physical 
meanings. Indeed, this number is the timescale ratio of avalanche motion $(l / g)^{0.5}$ and diffusion of disequilibrium pore fluid pressure normal to the flow direction $\left(\mu_{f} h^{2} / k E\right)$. Small values of $N_{P}$ indicate that high pore fluid pressure can be sustained quite long inside granular body and influence the avalanche motion significantly (Iverson et al. 2004). With a suggested hydraulic permeability $\left(k=10^{-11} \mathrm{~m}^{2}\right)$ and a compressive stiffness $\left(E=10^{7} \mathrm{~Pa}\right)$ for typical loose granular soils (Iverson 1997; Iverson et al. 2004), the $N_{P}$ values for surge and continuous debris flows can be calculated.

\subsection{Identification of surge and continuous debris flows}

We can calculate our dimensionless numbers (e.g., the grain Reynolds number and $N_{P}$ number) of the natural debris flows at DDFORS by applying the material parameters listed in Table 1 and the field observation data.

TABLE 1. Material parameters of the debris flows at DDFORS

\begin{tabular}{lc}
\hline Parameter & Value \\
\hline Unit weight of water, $\rho_{w}$ & $1000 \mathrm{~kg} / \mathrm{m}^{3}$ \\
Unit weight of solids, $\rho_{S}$ & $2750 \mathrm{~kg} / \mathrm{m}^{3}$ \\
Solids mean particle diameter, $d$ & $5 \mathrm{~mm}$ \\
Viscosity of pore fluid, $\mu_{f}$ & $0.5 \mathrm{~Pa} \cdot \mathrm{s}$ \\
Hydraulic permeability, $k$ & $10^{-11} \mathrm{~m}^{2}$ \\
Compressive stiffness for typical loose & $10^{7} \mathrm{~Pa}$ \\
granular soils, $E$ & \\
\hline
\end{tabular}

The dimensionless numbers values and the correlation between $N_{P}$ and contact behaviour of solid particles in natural debris flows is shown in Figs. 2a and 2b. Obviously, most of the grain Reynolds numbers $N_{\text {Rey }}$ are smaller than 1. This implies that the solid inertial stress is less dominant than the fluid viscous shearing stress. It also indicates that, for natural debris flows, fluid viscous shearing has a more significant effect on the mobility of the debris flows than do solid collisions. In contrast, if the pore fluid viscosity is small (e.g., 0.05 $\mathrm{Pa} \cdot \mathrm{s}$ suggested by Iverson (1997)), $N_{R e y}$ is greatly larger than 1 and the particle inertial stress may be dominant. In addition, when the grain Reynolds number is reduced, the roles played by viscous drag forces on the solid particles are more and more important. Moreover, the increase trend of the grain Reynolds number $N_{R e y}$ with $N_{P}$ implies that when solid particles have strong collisions and the debris flows tend to be inertial flows, pore fluid pressure in granular body will dissipate quickly and has little influence on particle contact behaviour. However, what should be emphasized here is that debris-flow behaviour is also significantly governed by solids concentration and it appears solids contact can be negligible at solid fractions of $30 \%$ even at a grain Reynolds number about 10, perhaps higher [4]. Different stress mechanisms, including those due to surface tractions (viscous drag), acceleration, the velocity fluctuations (Reynolds stress), and differences in density of solids and liquid (effective stress), should be considered to explain the behaviour of inertial flow of particle-laden fluids. Otherwise, it may neglect significant effects of other particle-scale momentum transport mechanisms except solids collisions.

Generally, the $N_{P}$ values of natural debris flows in DDFORS can be classified into three different zones. Most of the continuous flows possess high values of $N_{P}$ and focus in zone $1\left(N_{P}>0.03\right)$. In contrast, most of the surge flows have relatively small values of $N_{P}$ and focus in zone $3\left(N_{P}<0.01\right)$. Fig. $2 \mathrm{~b}$ shows that surge flows usually possess relatively larger solid concentrations than continuous flows. Due to the high solid concentration and the large traveling velocity for surge flows, high pore pressures are more likely developed in these flows. Relatively small $N_{P}$ values for surge flows indicate that the high pore fluid pressures can sustain quite long. Compared to surge flows, continuous flows with diluted solid particles possess high $N_{P}$ values. This implies that high pore pressures developed inside the granular body may dissipate quickly, the effects of pore fluid pressure are likely negligible.

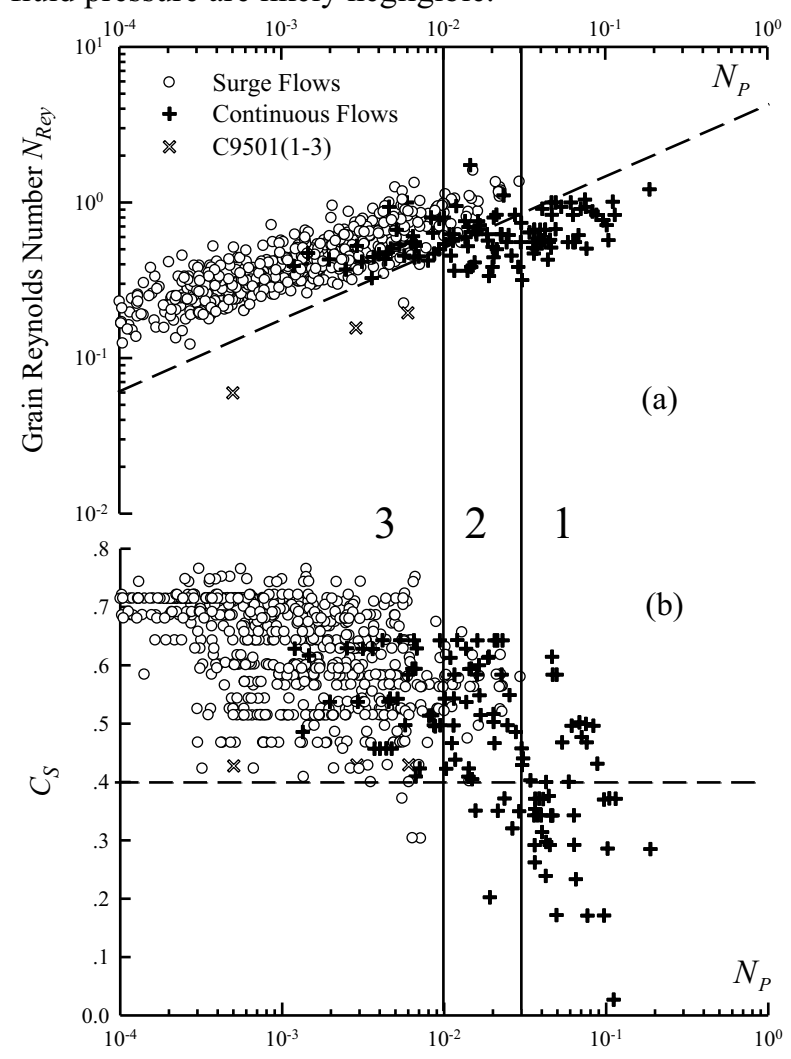

Fig. 2. Classification of natural debris flows as a function of dimensionless numbers: (a) the relationship between $N_{P}$ and grain Reynolds number $N_{\text {Rey }}$; (b) the relationship between $N_{P}$ and solid concentration $C_{S}$.

It is also obviously shown in the Fig. 2 that an overlapping zone exists: in zone $2\left(0.01<N_{P}<0.03\right)$, continuous and surge debris flows have the same $N_{P}$ values. Some flows in zone 2 are really difficult to identify as they possess both the continuous and surge flow properties. For this reason, the zone 2 can be treated as a transition zone. Based on the variation of $N_{P}$ values with the dimensionless number $N_{R e y}$, in zone 1 and 3 , the boundaries (dash lines) of distinguishing continuous and surge flows are approximately determined. Different 
debris flows in zone 2 can be classified by the dash lines (see Figs. 2a and 2b). For instance, by comparing the flows in zone 1 and 3 of Figs. $2 a$ and $2 b$, the flows locating above the dash lines generally can be treated as surge flows. Comparatively, the flows locating below the dash lines are treated as continuous flows. By this method, the classification of natural debris flows can be obtained with little ambiguity as different flows focus in different zones.

Due to the limitations of observation and subjective judgment, some flows originally treated as "continuous flows" indeed locate in zone 3 above the dash lines (see Figs. $2 \mathrm{a}$ and $2 \mathrm{~b}$ ). In these flows, the solid contact behaviour, pore fluid viscous drag, and the relative significance of pore pressure developed in the granular body are quite similar to that of surge flows. In addition, Fig. $2 b$ shows that the solid concentrations of these flows are sometimes relatively high $(>0.6)$. These "continuous flows" should be considered as surge flows. It is also interesting to find that the first three "continuous flows" occurring in 1995 (C9501(1-3)) locate in zone 3 below the dash lines. Comparing to the surge flows, the surge durations of these flows are relatively long and the flowing velocity is relatively low. Similar to surge flows, the timescale of avalanche motion for these flows is relatively smaller than the timescale for diffusion of disequilibrium pore fluid pressure. The small $N_{P}$ values imply that pore pressure inside the granular body may play a significant role. Moreover, the relative small $N_{\text {Rey }}$ values (comparing to those surge flows above the dash line in Fig. 2a) further illustrate that the pore fluid viscous shearing stress dominates particles inertial stress due to solids collision. For these debris flows, both the pore fluid viscous drag and pressues play a key role on the dynamics.

\section{Conclusions}

By comparing different values of the dimensionless numbers $N_{R e y}$ and $N_{P}$, we can predict the rheological properties of debris flows. Using this analysis on field data collected at the Dongchuan Debris Flow Observation and Research Station (DDFORS), we determined a number of properties of natural debris flows occurring on a large scale:

(1) Small values of $N_{\text {Rey }}(<1)$ illustrate that the natural debris flows at DDFORS are dense granular systems dominated by fluid viscous shearing stress rather than by particle collisions.

(2) Based on the dimensionless numbers of $N_{P}$ and $N_{R e y}$, the continuous and surge debris flows can be identified. The surge flows with high solid concentrations have relatively small $N_{P}$ values. It indicates that for surge flows, the high pore fluid pressures generated in granular body dissipate quite slowly and may influence particle contact behaviour significantly. When the natural debris flows tend to be solid collision dominated, pore fluid pressure will dissipate quickly and play a relatively negligible role to solid contact behaviour.

\section{Acknowledgments}

The authors acknowledge financial support from the National Natural Science Foundation of China (grant no. 11672318), the Key Research Program of Frontier Sciences, Chinese Academy of Sciences (grant no. QYZDB-SSWDQC010), and the Youth Innovation Promotion Association Chinese Academy of Sciences.

\section{References}

1. R. M. Iverson, Reviews of Geophysics 35 (3), 245 (1997).

2. G. Johnson, K. R. Rajagopal, M. Massoudi, USDOE Pittsburgh Energy Technology Center, PA (USA), (1990).

3. R. M. Iverson, Mathematical geology, 25(8): 1027-1048 (1993).

4. H. Haddadi, J. F. Morris, Journal of Fluid Mechanics 749, 431-459 (2014).

5. P. Coussot, M. Meunier, Earth-Science Reviews, 40(3): 209-227 (1996)

6. P. Coussot, Journal of Hydraulic Research, 32(4): 535-559 (1994).

7. P. Coussot, D. Laigle, Houille Blanche,3: $44-49$ (1994). (in French)

8. J. E. Costa, Physical geomorphology of debris flows, in Developments and Applications of Geomorphology, edited by J. E. Costa and P. J. Fleisher, pp. 268-317, New York: Springer-Verlag (1984).

9. T. Takahashi, Debris Flow. IAHR Monograph Series. Rotterdam: Balkema (1991).

10. P. Coussot, J. M. Piau, Can. Geotechn. J., 32, 263-270 (1995).

11. R. A. Bagnold, Proceedings of the Royal Society of London. Series A, Mathematical and Physical Sciences, Vol. 225 ( No. 1160), pp. 49 (1954).

12. P. Cui, X. Q. Chen, Y. Y. Wang, K. H. Hu and Y. Li, "Jiangjia Ravine debris flows in south-western China", In Debris-flow Hazards and Related Phenomena,edited by M. Jakob and O. Hungr. Berlin: Springer, 2005.pp. 565-594.

13. J. Zhang and G. Xiong, Data collection of kinematic observation of debris flows in Jiangjia Ravine, Dongchuan, Yunnan (1987-1994), Beijing: Science Press, 1997.

14. Z. C. Kang, P. Cui, F. Q. Wei and S. F. He, Data collection of observation of debris flows in Jiangjia Ravine, Dongchuan Debris Flow Observation and Research Station (1961-1984), Beijing: Science Press, 2006.

15. Z. C. Kang, P. Cui, F. Q. Wei and S. F. He, Data collection of observation of debris flows in Jiangjia Ravine, Dongchuan Debris Flow Observation and Research Station (1995-2000), Beijing: Science Press, 2007. 